\title{
Overview of MicroRNA Biology
}

\author{
Ashley M. Mohr, $\mathrm{PhD}^{1}$ Justin L. Mott, MD, $\mathrm{PhD}^{1}$ \\ ${ }^{1}$ Department of Biochemistry and Molecular Biology, University of \\ Nebraska Medical Center, Omaha, Nebraska \\ Semin Liver Dis 2015;35:3-11.
}

\begin{abstract}
Address for correspondence Justin L. Mott, MD, PhD, Department of Biochemistry and Molecular Biology, University of Nebraska Medical Center, 985870 Nebraska Medical Center, Omaha, NE 68198-5870 (e-mail: justin.mott@unmc.edu).
\end{abstract}
Abstract
Keywords
- ribonucleoprotein
- long noncoding RNA
- RNAi
- microRNA
- alternatives to seed binding

In considering an overview of microRNA biology, it is useful to consider microRNAs as a part of cellular communication. At the simplest level, microRNAs act to decrease the expression of messenger RNAs that contain stretches of sequence complementary to the microRNA. This function can be likened to the function of endogenous or synthetic short interfering RNA. However, microRNA function is more complicated and nuanced than this "on-off" model would suggest. Further, many microRNA targets are themselves noncoding RNAs. In this review, the authors discuss the role of microRNAs in shaping the proteome of the cell in a way that is consistent with microRNA involvement in a highly regulated conversation, sensitive to outside influence and internal feedback.

\section{MicroRNAs Function as Part of a Ribonucleoprotein Complex}

MicroRNAs act as imperfect sequence guides to recruit a ribonucleoprotein (RNP) complex to the complementary RNA. Using this paradigm, microRNAs act in a similar manner to other RNA components of RNPs, namely to provide a sequence-specific binding component to allow the RNP to act on a particular target. This is conceptually similar to mRNA splicing, where small nuclear RNAs (U1, U2, and U4-6) act through complementarity to sequences at the splice and branch sites within the intron that determine mRNA splicing. Small nuclear RNAs are bound to proteins and the resulting RNP (snRNP) directs splicing. ${ }^{1}$ In the case of snRNPs, the recognition sequences for splicing are often approximately seven to eight nucleotides long and allow occasional sequence mismatches and bulges.

The microRNA RNP complex is called the RNA-induced silencing complex (RISC), and similar to snRNPs, the RISC uses a small RNA (microRNA) to direct sequence-specific recruitment of the RISC to its target RNA. For microRNA-dependent targeting, often approximately seven to eight bases of complementarity are sufficient (though longer stretches of complementarity can enhance binding). Again, bulges and G:U wobble pairing are often allowed. The word "silencing" in RISC can give the wrong impression for a complex that more often acts to dampen and fine-tune expression instead of allout silencing.

\section{On or Off}

Conceptually and mechanistically related to RNA interference (RNAi) and siRNA, microRNAs can direct repression of target genes. The mechanisms by which microRNAs mediate target repression are diverse. ${ }^{2}$ In some cases, the microRNA binds with complementarity in the seed region (nucleotides 2-8 of the microRNA) as well as base pairing in the central region (bases $\sim 9-12$ ) causing mRNA cleavage and subsequent degradation. This sequence-specific degradation depends on RNA hydrolysis resulting in robust silencing. ${ }^{3}$ Because this endonucleolytic cleavage occurs at the phosphoester bond joining the target nucleotides that are opposite the microRNA bases $10-11^{4}$ and mismatches in this region are not well tolerated with regard to cleavage, ${ }^{5}$ it has been generalized that microRNA-target interactions with high degrees of complementarity result in mRNA degradation, whereas microRNA-target interactions with less complementarity function through translational repression. This simplification overlooks the data showing that microRNAs can cause target degradation without cleaving the target opposite position $10-11 .^{6}$ Thus, many microRNA targets are degraded even though they lack extensive complementarity.

Terms like silencing and even targeting suggest that repression of the target by a microRNA is either "on" or "off." Generally, microRNAs do not act to completely silence their target genes,
Issue Theme miRNA in Liver Pathobiology, Diagnosis, and Therapy; Guest Editor, Gregory J. Gores, MD
Copyright $\odot 2015$ by Thieme Medical Publishers, Inc., 333 Seventh Avenue, New York, NY 10001, USA.

Tel: +1(212) 584-4662.
DOI http://dx.doi.org/ 10.1055/s-0034-1397344. ISSN 0272-8087. 
but rather decrease expression. ${ }^{7,8}$ Similarly, microRNAs themselves are sometimes considered in this binary model to either be on or off. Indeed, there are examples where microRNAs have remarkable tissue specificity (e.g., miR-122 in the liver or miR124 in the brain ${ }^{9}$ ). There are also instances of strong induction of microRNAs at specified developmental times (e.g., let-7 in Caenorhabditis elegans development ${ }^{10}$ ). However, more often microRNAs are expressed in multiple cell and tissue types and have different expression levels, but rarely fit the dichotomous scheme of on or off. ${ }^{9}$

\section{Decreasing Target Expression}

There are many reports of the mechanisms by which microRNAs reduce expression of their cognate target proteins. This can include RNA degradation as above. ${ }^{11}$ Also, induced decapping, induced deadenylation, altered cap protein binding, reduced ribosome occupancy, and sequestration of the mRNA from translational machinery are reported. These mechanisms are not mutually exclusive and some result in decreased mRNA levels, whereas others act only to decrease protein expression. The relative contribution of mRNA degradation and translational repression was tested using microarray and ribosome profiling assays; it was found that the majority of the microRNA effect was mediated through decreased target mRNA levels. ${ }^{12}$ The practical result of this observation is that mRNA levels can be used to indicate microRNA targeting. However, because the magnitude of change in mRNA level is small and not all targets showed decreased mRNA levels, the use of only mRNA profiling to determine microRNA targets may still miss relevant target genes.

A model where microRNAs cause decreased expression of their targets is well supported. However, not all functional microRNA interactions involve a reduction in the expression of the target gene. For example, miR-373 has sequence complementarity to the promoter sequence of both E-cadherin and cold-shock domain-containing protein C2 (CSDC2). Transfection to increase levels of mature miR-373 caused increased mRNA expression of E-cadherin and CSDC2 by increased promoter occupancy by RNA Pol II. ${ }^{13}$ Increased expression of TNF- $\alpha$ protein due to microRNA-mediated recruitment of RISC to the AU-rich element in the $3^{\prime}$ untranslated region (3'UTR) of the TNF- $\alpha$ mRNA during cell cycle arrest was reported, suggesting that microRNAs can have stimulatory effects on expression depending on the timing within mitosis. ${ }^{14}$ Further, repression of the miR-122 target gene cationic amino acid transporter- 1 (CAT1) was reversed in cells subjected to stress by amino acid depletion, thapsigargin, or arsenite treatment. ${ }^{15}$ Binding of RISC to the target mRNA has the potential to displace other repressive RNA binding proteins and through this type of mechanism miR4661 increased the expression of the cytokine IL-10. The IL-10 mRNA, like many cytokine mRNAs, is targeted for degradation by proteins, such as tristetraprolin (TTP), that bind the AU-rich elements (AREs) located in the IL-10 3'UTR (untranslated region). ${ }^{16}$ IL-10 expression was thus increased by miR-466l outcompeting TTP binding at the 3'UTR of IL-10. Loss of TTP-directed degradation resulted in microRNA-directed increase in IL-10 expression. ${ }^{17}$ The predominant mode of microRNA function remains to decrease expression of targets with microRNA binding sites in the 3'UTR, but these reports demonstrate that under certain cellular conditions, the repressive function of microRNAs can be overcome; microRNA binding to the promoter can even increase target expression.

\section{Finding microRNA Targets}

Sequence complementarity between the microRNA and its target is preferentially located at the $5^{\prime}$ end of the microRNA, termed the seed. ${ }^{18}$ The seed consists of nucleotides $2-8$, counting from the microRNA 5' end. Complementarity at the 6-mer site from nucleotides 2-7 is generally not sufficient for target repression, but one base of additional complementarity at position 8 decreased target expression. ${ }^{19}$ The definition of the canonical seed binding site consisting of an A at position 1 followed by complementary bases over the subsequent seven positions is useful for predicting microRNA targets, ${ }^{18}$ but there are many functional targets that lack the canonical seed binding site. These include targets with G:U wobble pairing or bulges within the seed, illustrated by one of the original let-7 binding sites in lin-41. ${ }^{10} \mathrm{~A}$ particular type of pivoting at the seed-binding site resulting in a $\mathrm{G}$ bulge in the seed-binding site for miR-124 was even found to be a preferred microRNA target. ${ }^{20}$ Other target sites lack the seed-binding site altogether and instead rely on centered pairing. ${ }^{21}$ Recently, five classes of microRNA binding sites were described using data from many microRNA binding events. Class I sites rely only on the seed, class II sites showed seed binding and complementarity in nucleotides 13-16, and class III sites had seed-binding plus complementarity at nucleotides 17-21. Class IV sites lack seed binding and resemble centered pairing; class $\mathrm{V}$ sites had distributed or less stable binding. ${ }^{22}$ The concept of the seed remains a useful platform for understanding microRNA function, but additional or alternative points of binding are also important.

\section{Cellular Context}

A frustrating aspect of microRNA research is the ability of a single microRNA to have opposing functions in different systems, illustrating microRNA communication is context dependent. One example is miR-125b in cancer, downregulated in multiple cancers such as hepatocellular, breast, and lung while overexpressed in colorectal, pancreatic, gastric, and some leukemias. ${ }^{23}$ These results indicate that miR-125b has both oncogenic and tumor suppressive ability subject to the tissue/environment. This discrepancy can be partially explained by the targets of miR-125b, like p53. In certain cancer tissues, overexpression of miR-125b results in a loss of p53, blocking apoptosis. In other tissues, p53 may be mutated and miR-125b loss will allow expression of oncogenic targets, like epidermal growth factor receptor (EGFR) family members ERBB2/3 in breast cancer. ${ }^{23}$ Another study found the majority of microRNA targets in different cell types were not largely different, but were more different based upon differential 3' UTR isoforms and landscape. ${ }^{24}$ An additional concept in microRNA communication is how they interact together to target mRNAs. There are binding sites for many microRNAs in 
any given mRNA 3'UTR, allowing microRNAs to work together to increase repression of the target. In the pancreas, miR-375, miR-124, and let-7b were shown to work together to enhance myotrophin targeting. ${ }^{25}$ When the microRNAs are available to act in a coordinated manner, there is greater repression of the target mRNA.

\section{Regulation of microRNAs}

MicroRNAs are regulated by mechanisms similar to other RNAs, such as transcriptional activation or inhibition, epigenetic repression, and controlled degradation rates. Roughly half, $52 \%$, of human microRNAs are located in intergenic regions, $40 \%$ lie within intronic regions of genes, and the final $8 \%$ are exonic. ${ }^{26}$ Intronic microRNAs are often regulated by their host gene, and processed from the intron, but may have a distinct promoter region. Intergenic microRNAs typically have independent promoter elements. Upstream signaling initiates transcription of microRNA genes and may create feedback loops by targeting their own transcription factor(s). MiR-200c is involved in the epithelial-to-mesenchymal transition and is transcriptionally repressed by zinc finger E-box-binding homeobox 1 (ZEB1). ${ }^{27}$ However, if miR-200c is overexpressed it targets ZEB1 allowing for transcription, creating a forwardfeedback loop. This shows us that microRNAs can indirectly regulate their transcription. Processing of microRNAs by Drosha/DGCR8 in the nucleus creates precursor microRNAs with a 3' overhang. Dicer/TRBP in the cytoplasm recognizes this 3' overhang and further cleaves precursor forms to mature microRNAs. Both of these cleavage steps are reliant on the loop and secondary structure of the microRNA, differences in these alter processing. ${ }^{28}$ Further alterations, as described below, alter the processing efficiency and recognition of microRNAs. The half-life of microRNAs is generally long and many can persist for 5 days or longer; however, some microRNAs have rapid turnover. ${ }^{29}$ Multiple factors can account for the stability of microRNAs; some of which will be discussed below.

\section{MicroRNAs as Diagnostic Markers and Identifiers}

MicroRNA-expression profiles differ between disease states and normal tissue. Multiple studies have used microRNAs as diagnostics, either alone or in combination with other known biomarkers. Initial studies examining microRNA expression used tissues to determine functional and diagnostic roles of microRNAs. However, bodily fluids are more readily available and less invasive (in some instances) than biopsies. MicroRNAs are secreted by cells through exosomes and extracellular vesicles, ${ }^{30}$ and secreted microRNAs remain stable in bodily fluids. ${ }^{31}$ MicroRNAs have been isolated from blood (serum and plasma), saliva, urine, feces, follicular fluid, synovial fluid, pancreatic juice, bile, gastric juice, and other bodily fluids, and are being examined for utility as biomarkers for related diseases (-Fig. 1). A relevant example is microRNA profiling of bile to identify cholangiocarcinoma at an early stage. ${ }^{32}$ Bile from cholangiocarcinoma and control patients was assayed for the presence of microRNAs. They discovered a 5-micro-

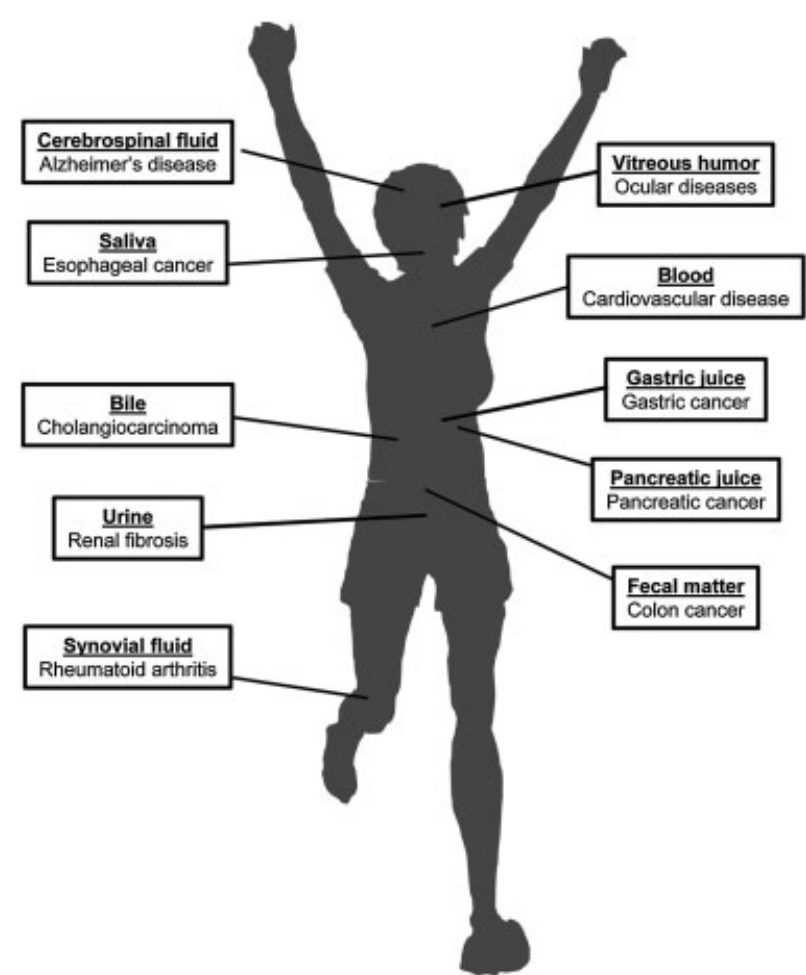

Fig. 1 MicroRNAs as biomarkers and prognostic factors. MicroRNAs are secreted into various bodily fluids that can be altered by disease states. Current studies are attempting to utilize microRNAs in these fluids for diagnostic and prognostic value. Examples are listed in this diagram: cerebrospinal fluid, Alzheimer disease ${ }^{1}$; vitreous humor, ocular diseases ${ }^{2}$; saliva, esophageal cancer $^{3}$; blood, cardiovascular disease $^{4}$; bile, cholangiocarcinoma ${ }^{5}$; gastric juice, gastric cancer ${ }^{6}$; pancreatic juice, pancreatic cancer ${ }^{7}$; urine, renal fibrosis ${ }^{8}$; fecal matter, colon cancer ${ }^{9}$; synovial fluid, rheumatoid arthritis ${ }^{10}$.

RNA panel that predicted early tumors better than carbohydrate antigen (CA19-9), with an overall sensitivity of 67\% and specificity of $96 \%$. Combining the microRNA panel with CA19-9, sensitivity increased to $89.7 \%$. This panel was also able to identify patients without metastatic lymph nodes better than CA19-9, indicating the ability to detect tumors at an early stage. MicroRNAs also have the ability to indicate the cell type being analyzed. The most well-known example of this is the liver-specific microRNA, miR-122. ${ }^{33}$ MiR-122 plays a role in cholesterol metabolism, hepatocellular carcinoma (HCC), and hepatitis $C$ virus infection. ${ }^{34}$ Other tissue-specific microRNAs include miR-134 and miR-124a in the brain, ${ }^{35}$ and miR-1 and miR-133 in the muscle. ${ }^{36}$

\section{MicroRNA Targets}

MicroRNAs have the potential to target hundreds of mRNAs due to the imperfect complementarity needed for binding. Indeed, RNA-sequencing for microRNA targets has identified hundreds of targets for a single microRNA. ${ }^{22}$ This creates a challenge identifying the functional role of microRNAs. One way to address the function of a microRNA, or family of microRNAs, is to determine a pathway or cellular function it most likely alters using predictive methods. MiR-21 has become well known in the cancer field and is overexpressed in the majority of human 
cancers. Its major role is inhibiting apoptosis, and miR-21 targets multiple proteins in both the intrinsic and extrinsic pathway leading to caspase activation. ${ }^{37,38}$ Another example of a microRNA affecting multiple proteins to fine-tune pathway function is observed in miR-14 signaling. Basal levels of miR-14 in Drosophila cells allow targeting of Hedgehog, a signaling protein involved in development, differentiation, and proliferation. ${ }^{39}$ However, increased miR-14 allows targeting of Patched and Smoothened, both members of the Hedgehog signaling pathway for a net decrease in Hedgehog signaling. This type of regulation by microRNA-expression levels permits either a passive voice (one or few targets) in cellular communication or a more dominant role (multiple targets in pathway).

So far, we have described microRNAs communicating in a single cell context; however, microRNAs have the ability to communicate with other cells or tissues. As discussed above, microRNAs are secreted into exosomes or microvesicles. These secreted microRNAs are very stable and can be taken up by cells in the surrounding tissue, or if the vesicles reached circulation, they can reach distant sites. ${ }^{40}$ MiR-150 was shown to be secreted by blood and monocytic cells and distributed to endothelial cells, conferring functional targeting of c-Myb. ${ }^{41}$ This is just one of many recent examples of how microRNAs communicate with their surroundings.

\section{MicroRNA Family Members}

MicroRNAs likely originated from duplication events, ${ }^{42}$ allowing for replication of identical or similar microRNAs. MicroRNAs that have highly similar sequence and secondary structure are considered to be family members. Because of these similarities, microRNAs in the same family often have overlapping targets, allowing for more robust repression of target pathways. The miR-17 92 cluster is a polycistronic microRNA that is comprised of six microRNAs: miR-17, -18 , $-19 a,-20,-19 b$, and -92 . This cluster is involved in development and is overexpressed in multiple cancers. ${ }^{43}$ The miR17 92 cluster has two paralogs, miR-106a 363 (miRs-106a, -18b, -20b, -19b-2, -92a-2, and -363) and miR-106b 25 (miRs106b, -93, and -25). Out of these paralogs (13 distinct microRNAs), there are four families: miR-17 family (miRs-17, -20a, -106a, -20b, -106b, and -93), miR-18 family (miRs-18a and -18b), miR-19 family (miRs-19a and b), and miR-92 family (miRs-92a, -363, and -25). Sequence similarity would propose similar function, however, it was found that miR-17 92, was necessary for mouse development; miR-106a 363 or miR$106 \mathrm{~b} \sim 25$ were not. However, deletion of both miR-17 92 and miR-106b 25 showed a more severe dysfunction in B cell development. ${ }^{44}$ This study showed that microRNA family members have individual and overlapping functions, broadening the complexity of microRNA function.

\section{Modified microRNAs}

Posttranscriptional modifications of microRNAs were once thought to be rare, however, it appears that these modified microRNAs (termed isomiR) are more common than once thought. Modifications of the 3 ' end of mRNAs alter the stability of the RNA, primarily by allowing for the decapping of the $5^{\prime}$ end followed by exonuclease degradation. ${ }^{45}$ Modification of microRNAs has been shown to have multiple roles. Uridylation can occur on both precursor microRNAs (premiR) and mature microRNAs. One example is with Lin28, an RNA-binding protein known to regulate let-7. Lin28 was associated with oligouridylation on the 3 ' end of pre-let-7. Oligouridylation inhibited Dicer recognition and cleavage, signaling microRNA degradation. ${ }^{46}$ However, when pre-let7 was monouridylated, there was an increase in Dicer activity resulting in higher levels of mature let- $7 .{ }^{47}$ Monouridylation of some let-7 precursor microRNAs increases the single nucleotide 3' overhang to two nucleotides, allowing better recognition by Dicer. The addition of uridine is common among other microRNA family members as well. ${ }^{48}$ In addition to precursor modification, mature microRNAs can also have $3^{\prime}$ nucleotide additions. MiR-26 targets Il-6 through canonical microRNA binding of the 3'UTR. However, uridylation of miR26 a inhibits regulation of Il-6. ${ }^{49}$ In addition to $3^{\prime}$ uridylation, 3' adenylation is also present on microRNAs and has been shown to either stabilize or degrade microRNAs. Specifically in the liver, mature miR-122 is 3' adenylated by PAP-associated domain containing 4 (PAPD4), a poly(A) polymerase, which increases stability. ${ }^{50}$ However, when miR-21 is adenylated by PAP-associated domain containing 5 (PAPD5), it is degradated. ${ }^{51}$ Although these 3 ' modifications of the microRNA affect stability, they likely do not alter mRNA targeting.

Other RNA editing of primary microRNA transcripts as well as precursors occurs, like adenosine deamination to inosine. This alteration not only changes the nucleoside, but also the structure of the precursor microRNA; inosine preferentially base pairs with cytidine, while adenosine preferentially binds uridine, thus causing new mismatches and bulges. Alterations in the secondary structure of microRNAs has the ability to alter the way RNA-binding proteins may interact, potentially inhibiting Drosha or Dicer processing of the primary and precursor forms. ${ }^{52,53}$ For example, pri-miR-151 can have adenosine to inosine RNA editing that completely blocks recognition and cleavage by dicer. ${ }^{53}$ If the modified primary or precursor microRNA is processed to a mature microRNA and the base substitution is in the seed region (or other highly regulatory area of the microRNA), target specification may be altered. Similarly for miR-376, adenosines are deaminated in the seed region, the modified miR-376 can bind alternate targets. ${ }^{54}$

In addition to 3' modifications and deamination, the 5 ' end of microRNAs can also have alternatives. The $5^{\prime}$ end of microRNA is of great importance of the function of the majority of microRNAs as it contains the seed region. Trimming of the $5^{\prime}$ end allows for seed shifting and alteration of potential mRNA targets. Differential Drosha cleavage of pri-miR-142 results in multiple 5 ' shifts, $-1,+1$, and $+2 .{ }^{55}$ The alterations in the 5 ' end of miR-142 likely change the seed sequence and mRNA targets.

\section{Alternative Splicing and Polyadenylation of Target Transcripts}

MicroRNA-based regulation of gene expression commonly occurs through microRNA binding to the $3^{\prime}$ untranslated 
region of the target mRNA. The length of the 3'UTR is a feature of the target gene, but can be altered through the use of alternative cleavage and polyadenylation signals (PAS). The PAS generally consists of a hexanucleotide 5'AAUAAA, but single base variants are described. Commonly, after the termination codon a gene will have multiple AAUAAA-like sequences, allowing for alternative usage. Alternative polyadenylation can shorten or lengthen the 3'UTR depending on whether an upstream signal becomes active or if the canonical PAS is skipped and a downstream signal is employed (-Fig. 2). Thus, alternative polyadenylation can result in the loss or gain of microRNA binding sites, a concept with experimental support. ${ }^{56-58}$ Interestingly, the miR-21 precursor sequence is located downstream of the gene for vacuole membrane protein-1 (VMP1) on the same strand, a few hundred bases distal to the PAS. MiR-21 is expressed at elevated levels in cancer and has oncogenic effects. Recently, skipping of the VMP1 PAS and use of an alternate distal sequence was shown to increase miR-21 expression by including the pre-mir-21 sequence in the 3'UTR of VMP1. ${ }^{59}$

Similar to the inclusion or exclusion of microRNA binding sites through alternative polyadenylation, alternative splicing has the potential to alter both the coding and UTR sequences of target mRNAs and thus alternative splicing has the potential to change microRNA binding potential. Although alternative splicing is understood to alter the coding region of an mRNA, alternative usage of splice sites at the 3 ' end of the transcript can result in completely different 3'UTR sequences for otherwise related mRNAs. As an example, the gene LMNA encodes both Lamin A and Lamin C, with Lamin A resulting from alternative splicing that results in inclusion of two additional exons and a novel 3'UTR compared with Lamin C. The protein products are identical for most of the protein with only a short C-terminal extension on Lamin A distinguishing the two. However, because the mRNAs each have different 3'UTR sequences, only Lamin A is regulated by the brain-specific miR-9. ${ }^{60}$ MicroRNAs encoded within introns of either coding or noncoding host genes are processed without regard for splicing ${ }^{61}$ suggesting that alternative splicing would not necessarily alter microRNA expression. However, there are microRNAs that span the exon-intron splice site and are responsive to splicing. For example, miR-412 is one of several microRNAs encoded on the lncRNA Mirg ${ }^{62}$ and the pre-miR-412 stem loop overlaps a splice site. Splicing interrupts the stem-loop of miR-412 preventing processing to premir-412 resulting in decreased miR-412 expression. On the other hand, skipping of the exon maintains the stem-loop and allows processing and expression. Alternative splicing of this IncRNA has a direct effect on expression of the microRNAs it contains. ${ }^{63}$ Alternative polyadenylation or alternative splicing can both change the conversation by affecting the responsiveness of the target gene to microRNA binding (presence or absence of the binding site) or by changing the level of the microRNA itself (i.e., miR-21 and miR-412).

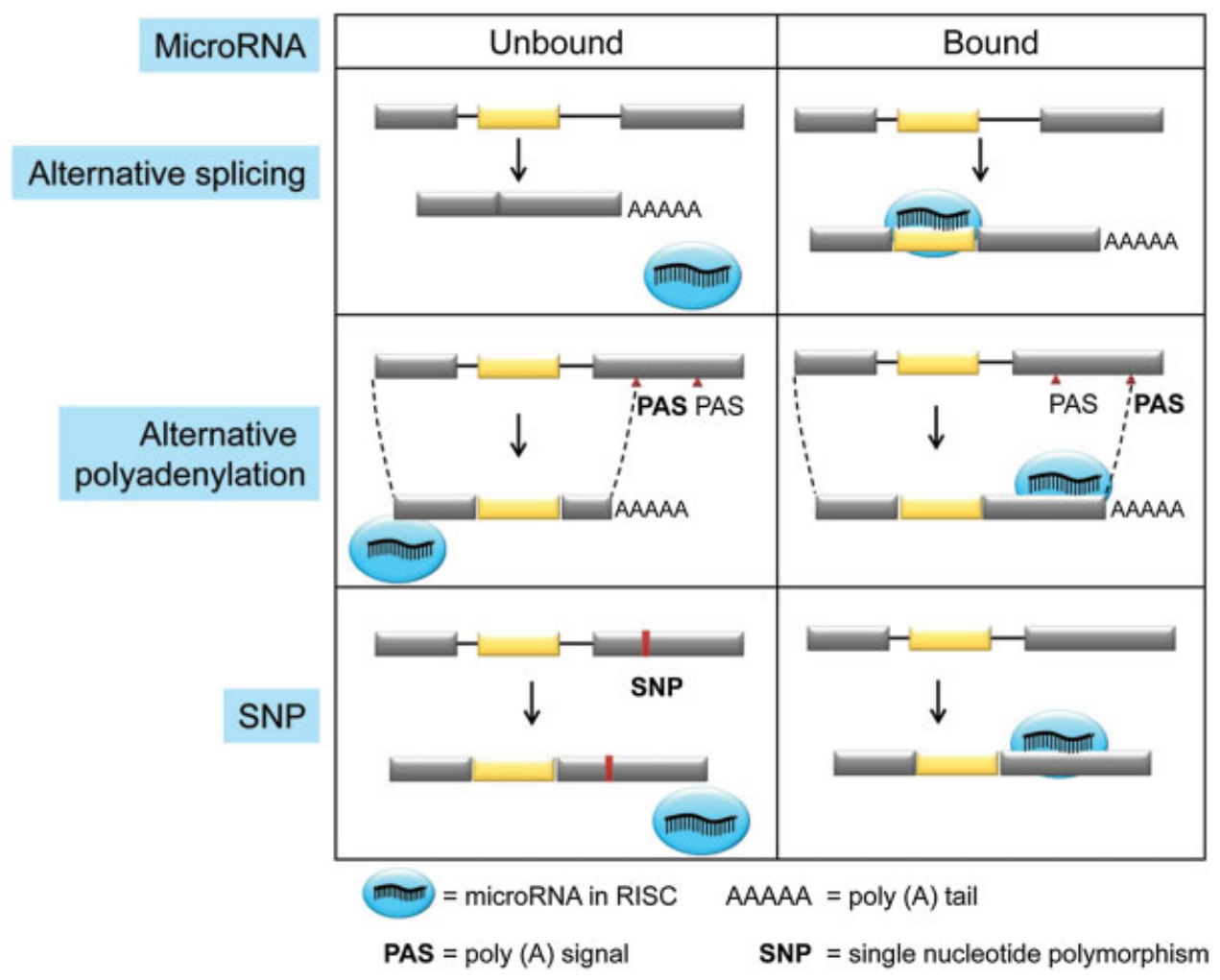

Fig. 2 Alternative microRNA targeting. Multiple instances may arise in which microRNAs can no longer bind to their intended targets. Three examples are (1) alternative splicing-the microRNA binding site is spliced out of the target transcript; (2) alternative polyadenylation signalsshort 3'UTRs lack the microRNA binding site; and (3) single nucleotide polymorphisms-altered microRNA binding sequence in the target mRNA inhibiting binding-site recognition. 


\section{Single-Nucleotide Polymorphisms}

Single nucleotide polymorphisms (SNPs) are relatively common base variances in which a base substitution is inherited; generally, there are only two alleles at the particular site: the common sequence and the SNP (present in $\sim 1-5 \%$ of individuals). Because SNPs are inherited and are physically linked to the neighboring genetic material, most do not change the cellular phenotype, but may be used as a marker of disease when a nearby gene carries a mutation (often the offending mutation is unknown and the SNP acts as a convenient means to infer the local change). However, several SNPs do change the function of their host gene; some also alter microRNA function or expression.

A single base change could impact microRNA function in several ways. For example, a SNP may unmask a cryptic microRNA binding site in the variant allele, resulting in a new functional interaction. Alternatively, if the variant alters a functional microRNA binding site, it may result in loss of microRNA-mediated regulation of the variant allele (-Fig. 2). In either case, for a patient with one of each allele, the result may be preferential expression of either the maternal or paternal allele of the gene.

An example of a disease-related SNP that regulates microRNA function by altering a microRNA binding site was found in the 3'UTR of KRAS. The allele resides in a binding site for let7 , and cells harboring a KRAS allele with the variant expressed higher levels of KRAS. Importantly, the variant was found in 18 to $20 \%$ of patients with nonsmall cell lung cancer, but only $6 \%$ of the general population. ${ }^{64}$ There are now bioinformatic resources to explore the effects of SNPs on microRNA sequence and binding sites. $^{65,66}$

\section{Regulation of Noncoding RNAs by microRNAs}

Considering the model of microRNAs as a sequence-specific guide to recruit the RISC to a target RNA, there is no a priori reason that the target must be a protein coding mRNA. MicroRNAs are one component of a class of RNA called noncoding RNAs (ncRNAs). In addition to microRNAs, there are many types of ncRNAs, including lncRNAs. Complementarity between microRNAs and other ncRNAs has been identified to be part of the conversation involving microRNAs and cell signaling. A few examples follow, with the note that there are more examples than those listed and systematic efforts are underway to catalog these interactions.

A direct functional relationship between lncRNAs and microRNAs was demonstrated previously in chronic lymphocytic leukemia. ${ }^{67}$ Recently, additional examples of microRNAs that directly bind to sequences in IncRNAs causing repression have been described. For example, metastasis associated lung adenocarcinoma transcript 1 (MALAT1) is expressed at increased levels in several cancer types and was found to be negatively correlated with miR-125b expression levels in bladder cancer. The effect was direct due to sequence complementarity between miR-125b and MALAT1, and the effects of increasing miR-125b were similar to the phenotype seen in cells with MALAT1 knockdown. ${ }^{68}$ Similarly, the noncoding RNA HCA1 is overexpressed in bladder cancer, whereas miR-1 is expressed at a lower level in this cancer. This inverse relationship suggests that miR-1 may repress HCA1 expression; indeed, there is a functional miR-1 binding site in HCA1. HCA1 repression was dependent upon Argonaute 2 expression, consistent with functional reduction of HCA1 by miR-1 through the RISC. ${ }^{69}$

Evidence that mature microRNAs in the RISC can bind to other mature microRNAs was captured using a technique called CLASH-cross-linking, ligation, and sequencing of hybrids. By immunopurifying RNAs bound to RISC followed by partial digestion and RNA-RNA ligation, the authors were able to identify microRNAs and their cognate targets through the production of chimeric RNA molecules. Noncoding RNAs made up $26 \%$ of the identified microRNA targets. One interaction highlighted was the binding of let-7 family members with mature miR-30b and miR-30c. Let-7 and miR-30 microRNAs have imperfect sequence complementarity to each other across most of their respective sequences (-Fig. 3 ). Additional ncRNA targets included tRNAs, rRNAs, and pseudogenes. ${ }^{22}$ In addition to mature microRNAs, let-7 can bind and regulate primary microRNA transcripts. Mature let-7, in complex with the $C$. elegans argonaut protein was identified in the nuclear fraction where it bound to unprocessed pri-let7. This interaction stimulated subsequent RNase-dependent processing of pri-let-7, resulting in a positive feedback role; let-7 functioned to further increase levels of mature let-7. ${ }^{70}$

Another example of ncRNA regulation of microRNA processing involves an lncRNA with an ultraconserved region and miR-195. Cleavage of pri-mir-195 by Drosha is regulated by Uc.283 + A. Uc.283 + A has a short (11 nucleotides) region of complementarity to pri-mir-195 at the sequence immediately upstream of the Drosha cleavage. Binding of Uc. $283+$ A to pri-miR-195 decreased microRNA processing presumably by altering the structural recognition of the stem-loop by the Drosha-containing microprocessor complex, resulting in decreased expression of mature miR-195 in the presence of Uc. $283+A .^{71}$ MicroRNAs can also affect the expression of IncRNAs indirectly. For example, miR-29 indirectly increased the expression of the IncRNA MEG3 in HCC by microRNAmediated reduction of the DNA methyltransferases DNMT1 and $3 \mathrm{~b}$, resulting in loss of epigenetic silencing of MEG3. ${ }^{72}$

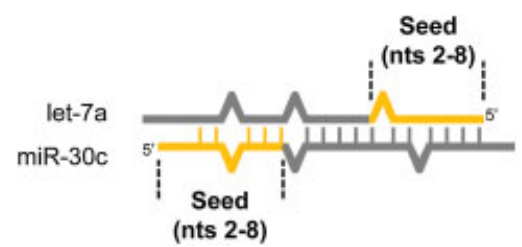

Fig. 3 MicroRNA-microRNA binding. Mature microRNAs can bind in a sequence-specific manner to each other, as demonstrated by crosslinking, ligation and sequencing of hybrids (CLASH). Illustrated here is the antiparallel microRNA duplex containing let-7a and miR-30c, adapted from Helwak et $\mathrm{al}^{22}$. The 5' terminus and seed region of each microRNA are indicated in yellow (nucleotides 1-8) and the remaining microRNA is in grey. Vertical "ladder rungs" indicate Watson-Crick base pairing, whereas the absence of a rung indicates a mismatch (i.e., within the miR-30c seed). Single nucleotide bulges (either symmetric or asymmetric) are depicted as a triangular deviation of the backbone. 
In conclusion, microRNAs can affect IncRNA expression directly or indirectly to alter cellular function. Not surprisingly, lncRNAs can also affect microRNA expression through sequence-specific binding that alters processing. Finally, microRNA-microRNA interaction in the RISC is reported. Short stretches of imperfect complementarity between microRNAs and their targets are sufficient to promote a functional interaction, suggesting that other interactions will be described. Known and predicted microRNA-lncRNA interactions are maintained in a searchable database, DIANALncBase. $^{73}$

\section{Conclusions}

MicroRNAs function as sequence-specific guides to direct a functional RNP to the target RNA of interest. When the target is a protein-coding mRNA, the most likely outcome of microRNA binding is decreased target protein expression. However, the effect is usually not to silence expression, but rather a more nuanced effect to decrease protein levels. This can be amplified by binding multiple microRNAs to a single target, or by the targeting of multiple proteins in the same pathway. When the target is a ncRNA, microRNA binding can also negatively affect expression of the target RNA. Similarly, ncRNAs can bind to the primary microRNA positively or negatively influencing processing and expression of the mature microRNA. We have begun to conceptualize microRNA function as a conversation within the cell. The discussion is generally not limited to a true/false or on/off effect of microRNAs, but is more like a drawn-out conversation. Expression of the microRNA itself is influenced by many participants in the conversation, at the level of transcription, processing, and function. Expression of the target RNA is likewise regulated at numerous levels by microRNAs, including epigenetic effects, promoter regulation, RNA processing and stability, and translation. The conversation was first noticed between microRNAs and protein-coding mRNAs, but is now recognized to be held between microRNAs and many other types of RNAs. Finally, the conversation is not limited to the cell where it began, as cell-cell transfer of microRNAs has functional effects on the downstream cell of interest. Just as in life, participants in the microRNA conversation come and go, whisper or shout, and have a positive or negative impact on others around them. We look forward to listening in as this cellular dialog continues; we are especially keen to learn how microRNAs can be used to steer the talk away from disease and toward health.

\section{Abbreviations}

$\begin{array}{ll}\text { 3'UTR } & \text { 3' untranslated region } \\ \text { ARE } & \text { AU-rich element } \\ \text { CA19-9 } & \text { carbohydrate antigen 19-9 } \\ \text { CAT1 } & \text { cationic amino acid transporter-1 } \\ \text { CSDC2 } & \begin{array}{l}\text { cold-shock domain-containing protein C2 } \\ \text { cross-linking, ligation and sequencing of }\end{array} \\ \text { CLASH } & \text { hybrids } \\ \text { HCC } & \text { hepatocellular carcinoma } \\ \text { IL } & \text { interleukin }\end{array}$

lncRNA long noncoding RNA

MALAT1 metastasis associated lung adenocarcinoma

$$
\text { transcript } 1
$$

ncRNA noncoding RNA

PAPD4/5 PAP-associated domain containing 4/5

PAS polyadenylation signal

pre-miRNA precursor microRNA

RNP ribonucleoprotein

RISC RNA-induced silencing complex

RNAi RNA interference

SiRNA short interfering RNA

SNP single nucleotide polymorphism

snRNP small nuclear RNP

TNF- $\alpha$ tumor necrosis factor alpha

TTP tristetraprolin

UTR untranslated region

VMP1 vacuole membrane protein-1

ZEB1 zinc finger E-box-binding homeobox 1

\section{Acknowledgments}

The project described was supported in part by the Nebraska Department of Health and Human Services (NE DHHS) through a Cancer and Smoking Disease Research Grant, funded by NE LB506. Additional support was from the Fred \& Pamela Buffett Cancer Center, University of Nebraska Medical Center, and from the National Institutes of Health, R03 DK092263. The contents of this manuscript are solely the responsibility of the authors.

\section{References}

1 Valadkhan S, Gunawardane LS. Role of small nuclear RNAs in eukaryotic gene expression. Essays Biochem 2013;54:79-90

2 Bartel DP. MicroRNAs: target recognition and regulatory functions. Cell 2009;136(2):215-233

3 Dykxhoorn DM, Novina CD, Sharp PA. Killing the messenger: short RNAs that silence gene expression. Nat Rev Mol Cell Biol 2003; 4(6):457-467

4 Elbashir SM, Martinez J, Patkaniowska A, Lendeckel W, Tuschl T. Functional anatomy of siRNAs for mediating efficient RNAi in Drosophila melanogaster embryo lysate. EMBO J 2001;20(23): 6877-6888

5 Martinez J, Tuschl T. RISC is a $5^{\prime}$ phosphomonoester-producing RNA endonuclease. Genes Dev 2004;18(9):975-980

6 Giraldez AJ, Mishima Y, Rihel J, et al. Zebrafish MiR-430 promotes deadenylation and clearance of maternal mRNAs. Science 2006; 312(5770):75-79

7 Baek D, Villén J, Shin C, Camargo FD, Gygi SP, Bartel DP. The impact of microRNAs on protein output. Nature 2008; 455(7209):64-71

8 Selbach M, Schwanhäusser B, Thierfelder N, Fang Z, Khanin R, Rajewsky N. Widespread changes in protein synthesis induced by microRNAs. Nature 2008;455(7209):58-63

9 Landgraf P, Rusu M, Sheridan R, et al. A mammalian microRNA expression atlas based on small RNA library sequencing. Cell 2007; 129(7):1401-1414

10 Reinhart BJ, Slack FJ, Basson M, et al. The 21-nucleotide let-7 RNA regulates developmental timing in Caenorhabditis elegans. Nature 2000;403(6772):901-906 
11 Meister G, Landthaler M, Patkaniowska A, Dorsett Y, Teng G, Tuschl T. Human Argonaute2 mediates RNA cleavage targeted by miRNAs and siRNAs. Mol Cell 2004;15(2):185-197

12 Guo H, Ingolia NT, Weissman JS, Bartel DP. Mammalian microRNAs predominantly act to decrease target mRNA levels. Nature 2010; 466(7308):835-840

13 Place RF, Li LC, Pookot D, Noonan EJ, Dahiya R. MicroRNA-373 induces expression of genes with complementary promoter sequences. Proc Natl Acad Sci U S A 2008;105(5):1608-1613

14 Vasudevan S, Tong Y, Steitz JA. Switching from repression to activation: microRNAs can up-regulate translation. Science 2007;318(5858):1931-1934

15 Bhattacharyya SN, Habermacher R, Martine U, Closs EI, Filipowicz $\mathrm{W}$. Relief of microRNA-mediated translational repression in human cells subjected to stress. Cell 2006;125(6):1111-1124

16 Brooks SA, Blackshear PJ. Tristetraprolin (TTP): interactions with mRNA and proteins, and current thoughts on mechanisms of action. Biochim Biophys Acta 2013;1829(6-7):666-679

17 Ma F, Liu X, Li D, et al. MicroRNA-466l upregulates IL-10 expression in TLR-triggered macrophages by antagonizing RNA-binding protein tristetraprolin-mediated IL-10 mRNA degradation. J Immunol 2010;184(11):6053-6059

18 Lewis BP, Burge CB, Bartel DP. Conserved seed pairing, often flanked by adenosines, indicates that thousands of human genes are microRNA targets. Cell 2005;120(1):15-20

19 Grimson A, Farh KK, Johnston WK, Garrett-Engele P, Lim LP, Bartel DP. MicroRNA targeting specificity in mammals: determinants beyond seed pairing. Mol Cell 2007;27(1):91-105

20 Chi SW, Hannon GJ, Darnell RB. An alternative mode of microRNA target recognition. Nat Struct Mol Biol 2012;19(3):321-327

21 Shin C, Nam JW, Farh KK, Chiang HR, Shkumatava A, Bartel DP. Expanding the microRNA targeting code: functional sites with centered pairing. Mol Cell 2010;38(6):789-802

22 Helwak A, Kudla G, Dudnakova T, Tollervey D. Mapping the human miRNA interactome by CLASH reveals frequent noncanonical binding. Cell 2013;153(3):654-665

23 Banzhaf-Strathmann J, Edbauer D. Good guy or bad guy: the opposing roles of microRNA 125b in cancer. Cell Commun Signal 2014; $12: 30$

24 Nam JW, Rissland OS, Koppstein D, et al. Global analyses of the effect of different cellular contexts on microRNA targeting. Mol Cell 2014;53(6):1031-1043

25 Krek A, Grün D, Poy MN, et al. Combinatorial microRNA target predictions. Nat Genet 2005;37(5):495-500

26 Hsu PW, Huang HD, Hsu SD, et al. miRNAMap: genomic maps of microRNA genes and their target genes in mammalian genomes. Nucleic Acids Res 2006;34(Database issue):D135-D139

27 Burk U, Schubert J, Wellner U, et al. A reciprocal repression between ZEB1 and members of the miR-200 family promotes EMT and invasion in cancer cells. EMBO Rep 2008;9(6):582-589

28 Michlewski G, Guil S, Semple CA, Cáceres JF. Posttranscriptional regulation of miRNAs harboring conserved terminal loops. Mol Cell 2008;32(3):383-393

29 Gantier MP, McCoy CE, Rusinova I, et al. Analysis of microRNA turnover in mammalian cells following Dicer1 ablation. Nucleic Acids Res 2011;39(13):5692-5703

30 Théry C. Exosomes: secreted vesicles and intercellular communications. F1000 Biol Rep 2011;3:15

31 Chen X, Ba Y, Ma L, et al. Characterization of microRNAs in serum: a novel class of biomarkers for diagnosis of cancer and other diseases. Cell Res 2008;18(10):997-1006

32 Li L, Masica D, Ishida M, et al. Human bile contains microRNAladen extracellular vesicles that can be used for cholangiocarcinoma diagnosis. Hepatology 2014;60(3):896-907

33 Lagos-Quintana M, Rauhut R, Yalcin A, Meyer J, Lendeckel W, Tuschl T. Identification of tissue-specific microRNAs from mouse. Curr Biol 2002;12(9):735-739
34 Jopling C. Liver-specific microRNA-122: Biogenesis and function. RNA Biol 2012;9(2):137-142

35 Schratt GM, Tuebing F, Nigh EA, et al. A brain-specific microRNA regulates dendritic spine development. Nature 2006;439(7074): 283-289

36 Nielsen S, Scheele C, Yfanti C, et al. Muscle specific microRNAs are regulated by endurance exercise in human skeletal muscle. J Physiol 2010;588(Pt 20):4029-4037

37 Buscaglia LE, Li Y. Apoptosis and the target genes of microRNA-21. Chin J Cancer 2011;30(6):371-380

38 Papagiannakopoulos T, Shapiro A, Kosik KS. MicroRNA-21 targets a network of key tumor-suppressive pathways in glioblastoma cells. Cancer Res 2008;68(19):8164-8172

39 Kim K, Vinayagam A, Perrimon N. A rapid genome-wide microRNA screen identifies miR-14 as a modulator of Hedgehog signaling. Cell Reports 2014;7(6):2066-2077

40 Valadi H, Ekström K, Bossios A, Sjöstrand M, Lee JJ, Lötvall JO. Exosome-mediated transfer of mRNAs and microRNAs is a novel mechanism of genetic exchange between cells. Nat Cell Biol 2007; 9(6):654-659

41 Zhang Y, Liu D, Chen X, et al. Secreted monocytic miR-150 enhances targeted endothelial cell migration. Mol Cell 2010; 39(1):133-144

42 Yuan Z, Sun X, Liu H, Xie J. MicroRNA genes derived from repetitive elements and expanded by segmental duplication events in mammalian genomes. PLoS ONE 2011;6(3):e17666

43 Olive V, Jiang I, He L. mir-17-92, a cluster of miRNAs in the midst of the cancer network. Int J Biochem Cell Biol 2010;42(8):1348-1354

44 Ventura A, Young AG, Winslow MM, et al. Targeted deletion reveals essential and overlapping functions of the miR-17 through 92 family of miRNA clusters. Cell 2008;132(5):875-886

45 Rissland OS, Norbury CJ. Decapping is preceded by 3' uridylation in a novel pathway of bulk mRNA turnover. Nat Struct Mol Biol 2009; 16(6):616-623

46 Heo I, Joo C, Cho J, Ha M, Han J, Kim VN. Lin28 mediates the terminal uridylation of let-7 precursor MicroRNA. Mol Cell 2008; 32(2):276-284

47 Heo I, Ha M, Lim J, et al. Mono-uridylation of pre-microRNA as a key step in the biogenesis of group II let-7 microRNAs. Cell 2012; 151(3):521-532

48 Newman MA, Mani V, Hammond SM. Deep sequencing of microRNA precursors reveals extensive 3 ' end modification. RNA 2011; 17(10):1795-1803

49 Jones MR, Quinton LJ, Blahna MT, et al. Zcchc11-dependent uridylation of microRNA directs cytokine expression. Nat Cell Biol 2009;11(9):1157-1163

50 Katoh T, Sakaguchi Y, Miyauchi K, et al. Selective stabilization of mammalian microRNAs by 3' adenylation mediated by the cytoplasmic poly(A) polymerase GLD-2. Genes Dev 2009;23(4): 433-438

51 Boele J, Persson H, Shin JW, et al. PAPD5-mediated 3' adenylation and subsequent degradation of miR-21 is disrupted in proliferative disease. Proc Natl Acad Sci U S A 2014;111(31): 11467-11472

52 Yang W, Chendrimada TP, Wang $\mathrm{Q}$ et al. Modulation of microRNA processing and expression through RNA editing by ADAR deaminases. Nat Struct Mol Biol 2006;13(1):13-21

53 Kawahara Y, Zinshteyn B, Chendrimada TP, Shiekhattar R, Nishikura K. RNA editing of the microRNA-151 precursor blocks cleavage by the Dicer-TRBP complex. EMBO Rep 2007;8(8): 763-769

54 Kawahara Y, Zinshteyn B, Sethupathy P, Iizasa H, Hatzigeorgiou AG, Nishikura K. Redirection of silencing targets by adenosine-toinosine editing of miRNAs. Science 2007;315(5815):1137-1140

$55 \mathrm{Wu} \mathrm{H}$, Ye C, Ramirez D, Manjunath N. Alternative processing of primary microRNA transcripts by Drosha generates 5 ' end variation of mature microRNA. PLOS ONE 2009;4(10):e7566 
56 Mayr C, Bartel DP. Widespread shortening of 3'UTRs by alternative cleavage and polyadenylation activates oncogenes in cancer cells. Cell 2009;138(4):673-684

57 Tranter M, Helsley RN, Paulding WR, et al. Coordinated posttranscriptional regulation of Hsp70.3 gene expression by microRNA and alternative polyadenylation. J Biol Chem 2011;286(34): 29828-29837

58 Boutet SC, Cheung TH, Quach NL, et al. Alternative polyadenylation mediates microRNA regulation of muscle stem cell function. Cell Stem Cell 2012;10(3):327-336

59 Ribas J, Ni X, Castanares M, et al. A novel source for miR-21 expression through the alternative polyadenylation of VMP1 gene transcripts. Nucleic Acids Res 2012;40(14):6821-6833

60 Jung HJ, Coffinier C, Choe Y, et al. Regulation of prelamin A but not lamin C by miR-9, a brain-specific microRNA. Proc Natl Acad Sci US A 2012;109(7):E423-E431

61 Kim YK, Kim VN. Processing of intronic microRNAs. EMBO J 2007; 26(3):775-783

62 Tierling S, Dalbert S, Schoppenhorst S, et al. High-resolution map and imprinting analysis of the Gtl2-Dnchc1 domain on mouse chromosome 12. Genomics 2006;87(2):225-235

63 Melamed Z, Levy A, Ashwal-Fluss R, et al. Alternative splicing regulates biogenesis of miRNAs located across exon-intron junctions. Mol Cell 2013;50(6):869-881

64 Chin LJ, Ratner E, Leng S, et al. A SNP in a let-7 microRNA complementary site in the KRAS $3^{\prime}$ untranslated region increases non-small cell lung cancer risk. Cancer Res 2008;68(20):8535-8540

65 Bhattacharya A, Ziebarth JD, Cui Y. PolymiRTS Database 3.0: linking polymorphisms in microRNAs and their target sites with human diseases and biological pathways. Nucleic Acids Res 2014;42 (Database issue):D86-D91

66 Bruno AE, Li L, Kalabus JL, Pan Y, Yu A, Hu Z. miRdSNP: a database of disease-associated SNPs and microRNA target sites on 3'UTRs of human genes. BMC Genomics 2012;13:44

67 Calin GA, Pekarsky Y, Croce CM. The role of microRNA and other non-coding RNA in the pathogenesis of chronic lymphocytic leukemia. Best Pract Res Clin Haematol 2007;20(3): 425-437

68 Han Y, Liu Y, Zhang H, et al. Hsa-miR-125b suppresses bladder cancer development by down-regulating oncogene SIRT7 and oncogenic long noncoding RNA MALAT1. FEBS Lett 2013; 587:3875-3882

69 Wang T, Yuan J, Feng N, et al. Hsa-miR-1 downregulates long noncoding RNA urothelial cancer associated 1 in bladder cancer. Tumour Biol 2014 [e-pub ahead of print]

70 Zisoulis DG, Kai ZS, Chang RK, Pasquinelli AE. Autoregulation of microRNA biogenesis by let-7 and Argonaute. Nature 2012; 486(7404):541-544

71 Liz J, Portela A, Soler M, et al. Regulation of pri-miRNA processing by a long noncoding RNA transcribed from an ultraconserved region. Mol Cell 2014;55(1):138-147

72 Braconi C, Kogure T, Valeri N, et al. microRNA-29 can regulate expression of the long non-coding RNA gene MEG3 in hepatocellular cancer. Oncogene 2011;30(47):4750-4756

73 Paraskevopoulou MD, Georgakilas G, Kostoulas N, et al. DIANALncBase: experimentally verified and computationally predicted microRNA targets on long non-coding RNAs. Nucleic Acids Res 2013;41(Database issue):D239-D245 